Schmerz 2009 $23: 565-566$

DOI 10.1007/s00482-009-0873-7

Online publiziert: 15. November 2009

(c) Deutsche Gesellschaft zum Studium

des Schmerzes. Published by Springer

Medizin Verlag - all rights reserved 2009

\author{
L. Radbruch ${ }^{1}$ H.-G. Schaible ${ }^{2}$ \\ ${ }^{1}$ Klinik für Palliativmedizin, RWTH Aachen \\ ${ }^{2}$ Institut für Physiologie I, Universitätsklinikum Jena
}

\section{Schmerz, Leid und Mitleid} Anleitung zum Umgang mit dem Tod
Die Entwicklung der modernen Medizin führt dazu, dass heute viele Erkrankungen geheilt oder zumindest für längere Zeit eingedämmt werden können, die noch vor wenigen Jahren schnell zum Tod geführt hätten. Andererseits wird häufig beklagt, dass dieser Fortschritt der modernen Medizin durch zunehmende Technisierung die Wahrnehmung des kranken Menschen hinter der Behandlung der Erkrankung zurücktreten lässt.

Genau hier setzen Palliativmedizin und Hospizarbeit an. Kontrolle der körperlichen Symptome ist eine unabdingbare Voraussetzung in der Betreuung Schwerkranker und Sterbender, die psychosoziale und die spirituelle Begleitung nehmen aber einen gleich hohen Stellenwert ein.

\section{- Nach der Definition der Weltge- sundheitsorganisation ist die Aufgabe der Palliativmedizin die ganzheitliche und fürsorgliche Behandlung des Patienten [4].}

Dies setzt aber eine Bereitschaft der Behandler voraus, eine emotionale Nähe zum Patienten zuzulassen und nicht nur die grundlegenden Einstellungen und Wertvorstellungen des Patienten, sondern auch seine Gefühle wahrzunehmen. Es wäre sicher falsch zu fordern, dass die Behandler auch mit den Gefühlen des Patienten mitschwingen, also tatsächlich mit-leiden im wahren Sinn des Wortes. Demgegenüber ist Mit-Leid, verstanden als ganzheitliche Wahrnehmung des $\mathrm{Pa}$ tienten, wie er sich selbst in seinem Leid erlebt, sehr hilfreich in der Kommunikation mit Patienten und Angehörigen.
Dies bei sich selbst wahrzunehmen und einzuordnen ist zumindest bei den Mitarbeitern in spezialisierten Teams notwendig, um langfristig die Arbeitsfähigkeit zu erhalten. Geschieht dies nicht, sind Überlastung und Burnout-Syndrom vorprogrammiert.

Für Palliativstationen gelten diese Überlegungen in besonderem Maß. Ärzte und Pflegepersonal haben sich meist freiwillig und nach reiflicher Überlegung für die Arbeit auf der Palliativstation entschieden. Viele Mitarbeiter im multiprofessionellen Team sind hoch motiviert, voller Enthusiasmus und mit überdurchschnittlichem Engagement in der täglichen Arbeit. Mittlerweile beträgt die durchschnittliche Liegedauer auf den deutschen Palliativstationen weniger als 10 Tage [2]. Dennoch gelingt es oft, in dieser kurzen Zeit eine intensive und empathische Beziehung zum Patienten aufzubauen. Allerdings versterben $40 \%$ der Patienten während der Behandlung auf der Palliativstation. Die Frage liegt also nahe, wie Mitarbeiter im Behandlungsteam mit diesen Todesfällen umgehen.

Müller et al. [3] stellen in dieser Ausgabe die Ergebnisse ihrer Umfrage zu der Frage „Wie viel Tod verträgt das Team“ vor. Die Fragestellung scheint symbolisch gemeint, und der Leser mag zunächst keine klare Antwort erwarten, sondern eine philosophische Diskussion zur Belastung und zu Belastungsfaktoren. Für den Leser überraschend, können die Autoren jedoch mit der von ihnen gewählten Methodik eine klare Antwort liefern: Es sind durchschnittlich 4,4 Todesfälle pro Woche, die als kritische Zahl genannt werden. Sterben (unabhängig von der Zahl der be- treuten Patienten) mehr als 4 Patienten in einer Woche, sind auch bei einem guten Team eine übermäßige Belastung und vielleicht sogar eine Krise unvermeidlich, wenn nicht dagegen interveniert wird.

Dies mag zunächst überraschen, zeigt aber auch, dass die befragten Mitarbeiter diese Situation in ihrem Arbeitsalltag erlebt und dabei die Grenzen ihrer Belastbarkeit wahrgenommen haben. Dies bestätigt auch die Sinnhaftigkeit und Notwendigkeit dieser Untersuchung. Mit diesem Ergebnis, mehr noch aber mit den differenzierten Auswertungen zu be- und entlastenden Faktoren und Mechanismen sind nicht nur den Teammitgliedern selbst, sondern auch den Organisatoren nun Möglichkeiten an die Hand gegeben, effektiv in solchen Krisensituationen zu intervenieren.

Als häufigste Belastungsfaktoren wurden ein nicht erfüllter Anspruch der Palliativmedizin und die Beziehung zum Patienten genannt, wichtigste Schutzfaktoren waren das Team, Humor und Privatleben. Für Ärzte lag die kritische Zahl an Todesfällen niedriger als für Pflegende, vielleicht im Zusammenhang mit der höheren Belastung durch Verantwortung und Schuldgefühle. Männer gaben eine höhere kritische Zahl an Todesfällen als Frauen an, aber natürlich kann dies auch nur Ausdruck einer höheren Rate an sozial erwünschten Antworten sein. In den Teams, die mit Sprachlosigkeit oder mit Dienst nach Vorschrift auf den Tod reagierten, wurde eine niedrigere kritische Zahl angegeben.

Die Autoren betonen in ihren Schlussfolgerungen die Bedeutung der Kommunikation im Team sowie die Diskussion der 
eigenen Ansprüche an die Palliativmedizin im Team als Schutzfaktoren. In Ergänzung scheint uns die Diskussion des ärztlichen Selbstbildes und Rollenverständnisses notwendig, um Schuldgefühlen und der Belastung durch die Verantwortung zu begegnen.

Dies ist aller Wahrscheinlichkeit nach nicht nur für die Ärzte auf Palliativstationen von Bedeutung, sondern auch in anderen ambulanten und stationären Bereichen, in denen schwerkranke und sterbende Patienten betreut werden. In einem Seminar zur Begleitung von Palliativpatienten berichteten die Medizinstudenten an der RWTH Aachen, dass die Balance zwischen kurzzeitiger menschlicher Nähe und professioneller Distanz eine zentrale Frage in der eigenen Rollendefinition war [1].

Diese Fragen sollten deshalb sicher auch Teil der ärztlichen Ausbildung sein, für die im September 2009 mit dem Gesetz zur Regelung des Assistenzpflegebedarfs im Krankenhaus Palliativmedizin als Pflichtfach im Medizinstudium eingeführt worden ist.

\section{- Das neue Pflichtfach darf sich also nicht auf die Vermittlung von Kenntnissen zur Schmerzlinderung und Symptomkontrolle beschränken, sondern muss Impulse für Haltungen und Einstellungen der Studenten geben.}

Dies kann nicht durch Frontalunterricht erreicht werden, sondern es sind innovative Unterrichtskonzepte gefordert, wie sie an den Lehrstühlen für Palliativmedizin in Aachen, Bonn, Göttingen, Köln und München entwickelt werden.

Es bleibt allerdings $\mathrm{zu}$ fragen, wieweit diese Fragestellung als Thema für die Zeitschrift „Der Schmerz“ gelten darf. Im Manuskript wird Schmerz nur am Rande erwähnt. Als Herausgeber könnten wir aber argumentieren, dass die emotionale Belastung durch die Todesfälle zu Leid führt und dieses Leid einem emotionalen Schmerz beim Behandler entspricht. Wir haben uns jedoch aus einem anderen Grund für die Publikation des Beitrags entschieden.

Entscheidend war für uns die Überzeugung, dass die Zeitschrift „Der Schmerz“ sich nach den Interessen der Leser richten sollte und dass diese Interessen auch die Randgebiete der Schmerztherapie und die Schnittmengen mit nahestehenden Fächern umfassen.

\section{- Die inhaltliche Nähe von \\ Schmerztherapie und \\ Palliativmedizin, das palliativ- \\ medizinische Engagement vieler \\ Schmerztherapeuten und das \\ Interesse vieler Palliativmediziner \\ an der Schmerzforschung stellen \\ eine solche Schnittmenge dar.}

In der Vergangenheit wurden bereits vereinzelt ähnliche Beiträge mit eher indirektem Bezug zu Schmerz und Schmerztherapie in „Der Schmerz" veröffentlicht. So wurden in einem Beitrag aus unserer eigenen Abteilung der Einsatz von Benzodiazepinen und die Praxis der palliativen Sedierung in einer Palliativstation untersucht [5]. „Der Schmerz“ tritt damit nicht in Konkurrenz zu palliativmedizinischen Zeitschriften, sondern soll deren Angebot ergänzen und erweitern.

Nicht zuletzt wollen wir so ermöglichen, dass herausragende palliativmedizinische Themen Zugang zu einer in Medline aufgeführten deutschsprachigen Zeitschrift mit Impaktfaktor erhalten. Die Arbeit von Müller et al. erfüllt sicher diese Ansprüche, sie ist von hoher Qualität und von hohem Interesse für den Leser.
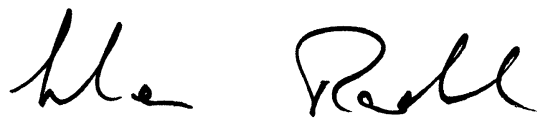

L. Radbruch

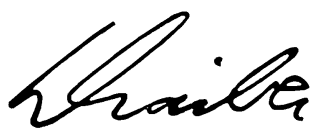

H.-G. Schaible

\section{Korrespondenzadresse}

Prof. Dr. L. Radbruch

Klinik für Palliativmedizin, RWTH Aachen

Pauwelssstr. 30, 52074 Aachen

LRadbruch@ukaachen.de

\section{Literatur}

1. Elsner $F$, Jünger $S$, Pestinger $M$, Krumm N, Radbruch L (2006) Der Patient in der Rolle des Lehrers. Z Palliativmed 7:131-135

2. Lindena G, Woskanjan S, Fahland R (2009) Hospizund Palliativerhebung 2009 Bericht. CLARA Clinical Analysis, Research and Application, Kleinmachnow

3. Müller M, Pfister DG, Markett S, Jaspers B (2009) Wie viel Tod verträgt ein Team. Schmerz (online first DOI 10.1007/s00482-009-0845-y)

4. Sepulveda C, Marlin A, Yoshida T, Ullrich A (2002) Palliative care: the World Health Organization's global perspective. J Pain Symptom Management 24:91-96

5. Stiel S, Krumm N, Schroers O, Radbruch L, Elsner F (2008) Indikationen und Gebrauch von Benzodiazepinen auf einer Palliativstation. Schmerz 22:665-671 